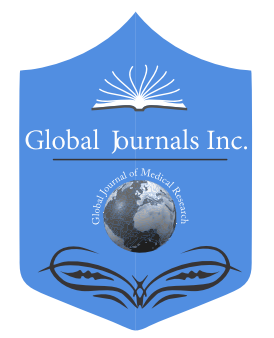

GLOBAL JOURNAL OF MEDICAL RESEARCH: B

PHARMA, DRUG DisCOVERY, TOXICOLOGY \& MEDICINE

Volume 21 Issue 3 Version 1.0 Year 2021

Type: Double Blind Peer Reviewed International Research Journal

Publisher: Global Journals

Online ISSN: 2249-4618 \& Print ISSN: 0975-5888

\title{
Evaluation De L'activité Anthelminthique Des Extraits Aqueux De Striga Hermonthica (Del.) Benth Et Ceratotheca Sésamoïdes Endl Sur Deux Stades Biologiques De Haemonchus Contortus
}

\author{
By Amadou Dicko, Almamy Konate, Arnaud S. R. Tapsoba, L. D. Dahourou, \\ Moumouni Sanou, Adama Kabore, S. Tembely, Linda L. Logan, Amadou Traore, \\ Balé Bayala \& Hamidou H. Tamboura
}

Abstract- Medicinal plants with anthelmintic properties are an alternative to the chemical fight against small ruminant's gastrointestinal nematodes. In order to broaden the spectrum of medicinal plants with anthelmintic properties, ovicidal activity and L3 larvae exsheathment of C. sesamoïdes Endl and S. hermonthica (Deli) Benth, aqueous extracts has been done on $H$ contortus egg and L3 larvae. Three concentrations, $3.12 \mathrm{mg} / \mathrm{ml}, 6.25 \mathrm{mg} / \mathrm{ml}, 12.5 \mathrm{mg} / \mathrm{ml}$ of each plant extract were used to assess the inhibition of fresh egg hatching and larval paralysis as well as the 'inhibition of L3 larvae exsheathment. A negative control (PBS1x) and a positive control (levamisole, $2.5 \mathrm{mg} / \mathrm{ml}$ ) were constituted for the fresh egg hatching inhibition assessment while only the negative control (PBS1x) was constituted to evaluate the inhibition of L3 Larvae exsheathment. $1 \mathrm{ml}$ of each concentration was contacted with $1 \mathrm{ml}$ of the egg solution and then incubated for 48 hours at $27^{\circ} \mathrm{C}$ in petri dishes $(60 \times 15 \mathrm{Cm})$ for hatching test inhibition. 1 $\mathrm{ml}$ of each extract concentration was contacted with $1 \mathrm{ml}$ of the embryonated egg solution after 24 hours of incubation at $27^{\circ} \mathrm{C}$ for the larval paralysis test.

Keywords: ceratothéca sesamoides endl, striga hermonthica (Deli.) benth, H. contortus, egg hatching inhibition, L3 larval exsheathment inhibition.

GJMR-B Classification: NLMC Code: QV 704

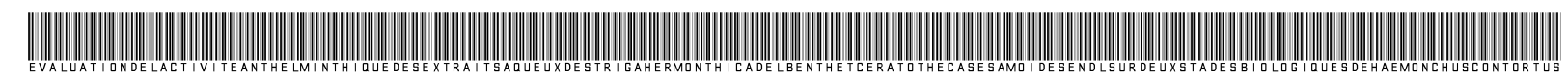

Strictly as per the compliance and regulations of:

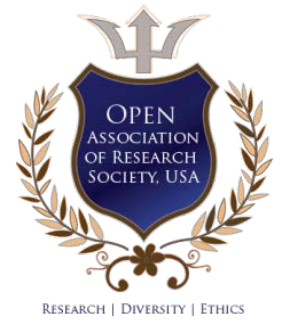

(c) 2021. Amadou Dicko, Almamy Konate, Arnaud S. R. Tapsoba, L. D. Dahourou, Moumouni Sanou, Adama Kabore, S. Tembely, Linda L. Logan, Amadou Traore, Bale Bayala \& Hamidou H. Tamboura. This research/review article is distributed under the terms of the Attribution-NonCommercial-NoDerivatives 4.0 International (CC BY-NC-ND 4.0). You must give appropriate credit to authors and reference this article if parts of the article are reproduced in any manner. Applicable licensing terms are at https://creativecommons.org/licenses/by-nc-nd/4.0/. 


\title{
Evaluation De L'activité Anthelminthique Des Extraits Aqueux De Striga Hermonthica (Del.) Benth Et Ceratotheca Sésamoïdes Endl Sur Deux Stades Biologiques De Haemonchus Contortus
}

\author{
Amadou Dicko ${ }^{\alpha}$, Almamy Konate $^{\sigma},{\text { Arnaud S. R. } \operatorname{Tapsoba}^{\circ}, \text { L. D. Dahourou }}^{\omega}$, Moumouni Sanou $^{*}$, \\ Adama Kabore ${ }^{\S}$, S. Tembely ${ }^{\circledR}$, Linda L. Logan ${ }^{\vee}$, Amadou Traore ${ }^{\ominus}$, Balé Bayala ${ }^{\S}$ \\ \& Hamidou H. Tamboura ${ }^{\mathrm{f}}$
}

Résumé- Les plantes médicinales à propriétés anthelminthiques constituent une alternative à la lutte chimique contre les nématodes gastro-intestinaux des petits ruminants. Dans l'objectif d'élargir le spectre des plantes médicinales à propriétés anthelminthiques, une évaluation de l'activité ovicide et d'inhibition du dégainement des larves L 3 de $H$ contortus, des extraits aqueux de $C$. sesamoïdes Endl et $S$. hermonthica (Deli.) Benth a été effectuée. Trois concentrations, $3,12 \mathrm{mg} / \mathrm{ml}, 6,25 \mathrm{mg} / \mathrm{ml}, 12,5 \mathrm{mg} / \mathrm{ml}$ de chaque extrait de plante ont été utilisées pour évaluer l'inhibition de l'éclosion des œufs frais et de paralysie larvaire ainsi que l'inhibition du dégainement des larves L3. Un Témoin négatif (PBS1x) et un témoin positif (lévamisole, $2,5 \mathrm{mg} / \mathrm{ml}$ ) ont été constitués pour l'évaluation de l'inhibition de l'éclosion alors que seulement le témoin négatif (PBS1x) a été constitué pour évaluer l'inhibition du dégainement des larves $L 3.1 \mathrm{ml}$ de chaque concentration a été mise en contact avec $1 \mathrm{ml}$ de la solution d'œufs puis incuber pendant 48 heures à $27^{\circ} \mathrm{C}$ dans des boîtes de pétri $(60 \times 15 \mathrm{Cm})$ pour le test de l'inhibition de l'éclosion. $1 \mathrm{ml}$ de chaque concentration d'extrait a été mise en contact avec $1 \mathrm{ml}$ de la solution d'œuf embryonné après 24 heures d'incubation à $27^{\circ} \mathrm{C}$ pour le test sur la paralysie larvaire. $1 \mathrm{ml}$ de la solution larvaire a été mise en contact avec $4 \mathrm{ml}$ de chaque concentration de la solution d'extrait pendant 3 heures pour l'inhibition du dégainement des larves L3. Les résultats ont montré une inhibition de l'éclosion de $53,89 \%$ à la concentration de $12,5 \mathrm{mg} / \mathrm{ml}$ avec $C$. sesamoïdes Endl approximativement au témoin positif (Lévamisole) $56,49 \%$ alors que celui du $S$. hermonthica n'a été que de $40,72 \%$. Le taux de paralysie larvaire a été de $51,11 \%$ et $60,56 \%$ respectivement pour $S$.hermonthica (Deli.) Benth et

Author $\alpha \sigma \rho ¥ \oplus f$ : Laboratoire de Biologie et Santé Animale (LaBioSA), Institut de l'Environnement et de Recherches Agricoles (INERA) 04BP 8645, Ouagadougou 04 Burkina Faso.

e-mail: dicko.amadou36@yahoo.fr

Author z: Laboratoire de Physiologie Animale (LaPA), Unité de Formation et de Recherches en Sciences de la vie et de la Terre. Université OUAGA I Joseph KI-ZERBO, O3 BP 7021, Ouagadougou 03, Burkina Faso,

Author v: College of Veterinary Medicine and Biomedical Sciences, Texas A\&M University, 660 Raymond Stotzer Pkwy, College Station, TX 77843, USA.

Author X: Académie des Sciences du Mali, Baco-Djicoroni ACl Ouest, Rue 619 Porte, 104 Bamako, Mali.

Author $\omega$ : Institut des Sciences de l'Environnement et du Développement Rural (ISEDR), Université de Dédougou, Dédougou BP 176, Burkina Faso.
C. sesamoïdes Endl. L'inhibition du dégainement pour l'extrait aqueux de $C$. sesamoïdes Endl est de $65,33 \%$ à $12,5 \mathrm{mg} / \mathrm{ml}$ et $64 \%$ à la même concentration pour $S$. hermonthica (Deli.) à Benth après 60 minutes.

Mots clés: ceratothéca sesamoides endl, striga hermonthica (Deli.) benth, $H$. contortus, inhibition éclosion œuf, inhibition dégainement larves $L 3$.

Abstract-Medicinal plants with anthelmintic properties are an alternative to the chemical fight against small ruminant's gastrointestinal nematodes. In order to broaden the spectrum of medicinal plants with anthelmintic properties, ovicidal activity and L3 larvae exsheathment of $C$. sesamoïdes Endl and $S$. hermonthica (Deli) Benth, aqueous extracts has been done on $H$ contortus egg and L3 larvae. Three concentrations, $3.12 \mathrm{mg} / \mathrm{ml}, 6.25 \mathrm{mg} / \mathrm{ml}, 12.5 \mathrm{mg} / \mathrm{ml}$ of each plant extract were used to assess the inhibition of fresh egg hatching and larval paralysis as well as the 'inhibition of L3 larvae exsheathment. A negative control (PBS1x) and a positive control (levamisole, $2.5 \mathrm{mg} / \mathrm{ml}$ ) were constituted for the fresh egg hatching inhibition assessment while only the negative control (PBS1x) was constituted to evaluate the inhibition of L3 Larvae exsheathment.1 $\mathrm{ml}$ of each concentration was contacted with $1 \mathrm{ml}$ of the egg solution and then incubated for 48 hours at $27^{\circ} \mathrm{C}$ in petri dishes $(60 \times 15 \mathrm{Cm})$ for hatching test inhibition. $1 \mathrm{ml}$ of each extract concentration was contacted with $1 \mathrm{ml}$ of the embryonated egg solution after 24 hours of incubation at $27^{\circ} \mathrm{C}$ for the larval paralysis test. $1 \mathrm{ml}$ of the larval solution was contacted with $4 \mathrm{ml}$ of each concentration of the extract solution for L3 larvae exsheathment inhibition test. The results showed a hatching inhibition of $53.89 \%$ at the concentration of $12.5 \mathrm{mg} \mathrm{/} \mathrm{ml}$ with $C$. sesamoids Endl aproximatly to the positive control (Levamisole) $56.49 \%$ whereas hatching inhibition of $S$. hermonthica was only of $40.72 \%$. The larval paralysis rate was $51.11 \%$ and $60.56 \%$ respectively for $S$. hermonthica (Deli.) Benth and $C$. sesamoïdes Endl. The inhibition of L3 larvae exsheathment for the aqueous extract of $C$. sesamoides Endl is $65.33 \%$ at 12.5 $\mathrm{mg} / \mathrm{ml}$ and $64 \%$ at the same concentration for $S$. hermonthica (Deli.) Benth after 60 minutes.

Keywords: ceratothéca sesamoides endl, striga hermonthica (Deli.) benth, H. contortus, egg hatching inhibition, L3 larval exsheathment inhibition. 


\section{InTRODUCTION}

H aemonchus contortus est l'un des nématodes gastro-intestinaux qui cause la plus importante contrainte dans l'élevage des petits ruminants (Mangi et al, 2006). En effet, la faune parasitaire du tractus digestif des petits ruminants est dominée par $\mathrm{H}$. contortus de par sa fréquence et son intensité (Koné et al, 2005; Dedehou et al, 2014; Koffi et al, 2018). Comparativement aux autres espèces de nématodes, $H$. contortus est le parasite des petits ruminants le plus pathogène capable de provoquer des maladies aiguës et des fortes mortalités dans toutes les catégories des petits ruminants (Akkari et al, 2013 ; MbogningTayo et al, 2014). Ce parasite hématophage peut causer une anémie conduisant à la réduction de la productivité chez les petits ruminants (Burke et al, 2007).

La gestion des nématodes gastro-intestinaux des petits ruminants repose fortement sur l'utilisation des anthelminthiques de synthèses (Chartier et al, 2014). L'utilisation répétée de ces anthelminthiques de synthèse a entrainé l'apparition des résistances irréversibles des parasites à la majorité des familles des molécules anthelminthiques (Apala et al, 2020). Aussi, en plus de la difficulté d'accès de ces molécules anthelminthiques de synthèse aux éleveurs ruraux, il faut ajouter l'écotoxicité de ces molécules pour l'environnement (Dedehou et al, 2014).

Face à cette situation différentes alternatives aux molécules anthelminthiques dans le traitement des nématodes gastro-intestinaux sont envisagées. Parmi ces solutions alternatives il y a l'utilisation des plantes fourragères à propriétés anthelminthiques.

L'objectif de cette présente étude est d'évaluer in vitro l'activité ovicide et inhibitrice du dégainement des larves $L 3$ de $H$. contortus des extraits aqueux du Strigahermonthica (Del.) Benth et de C. sésamoïdes Endl.

\section{il. Matériel et Méthode}

\section{a) Milieu d'étude}

Les tests in vitrode l'évaluation de l'activité ovicide et inhibitrice du dégainement larvaire ont été réalisés au Laboratoire de Biologie et santé Animale (LaBioSA) du Centre de Recherches Environnementales, Agricoles et de Formation (CREAF) de Kamboinsin.

\section{b) Récoltes des plantes}

Cératothéca sésamoïdes Endl et Striga hermontica (Delile) Benth ont été récoltées puis séchées à l'ombre entre fin Septembre et mi-octobre dans une salle à la température ambiante à la Direction Régionale de la Recherche Environnementale et Agricoles (DRREA-Sahel). Les plantes ont été identifiées à l'Herbier National du Burkina Faso (HNBF) respectivement sous les numéros 8758 et 8759 .

\section{c) Préparation des extraits}

Les plantes entières des deux espèces ont été récoltées tôt le matin et séchées dans une salle à la DRREA-Sahel à la température ambiante pendant 2 semaines et acheminées au CREAF de Kamboinsin par la suite. Les échantillons de chaque plante ont été par la suite broyés en poudre. Une macération aqueuse (100 $\mathrm{g}$ de poudre dans $900 \mathrm{ml}$ d'eau distillée) a été effectuée pendant 24 heures sous agitation mécanique à la température ambiante. Les macérations ont été ensuite filtrées et concentrées au congélateur. Les filtrats concentrés ont été par la suite lyophilisées.

\section{d) Tests biologiques}

\section{i. Préparations des solutions mères}

Les solutions mères de chaque extrait ont été préparées en diluant $0,25 \mathrm{~g}$ d'extrait de chaque plante dans $10 \mathrm{ml}$ du PBS $1 \mathrm{X}$ pour obtenir $25 \mathrm{mg} / \mathrm{ml}$ de concentration pour chaque extrait. La solution a été homogénéisée à l'aide d'un sonicateur pendant 3 minutes. A partir de la solution mère trois autres solutions de concentrations inferieures qui seront utilisées à savoir $12,5 \mathrm{mg} / \mathrm{ml}$ et $6,25 \mathrm{mg} / \mathrm{ml}$ et 3,12 $\mathrm{mg} / \mathrm{ml}$ de chaque extrait ont été réalisées. Le lévamisole (anthelminthique de référence) à $2,5 \mathrm{mg} / \mathrm{ml}$ et PBS $1 \mathrm{X}$ ont été utilisé respectivement comme témoins positif et négatif.

\section{ii. Récoltes des œufs}

Les œufs ont été obtenu selon la méthode décrite par Kaboré. (2009). Tout d'abord, Les vers adultes ont été récoltés sur des caillettes des ovins fraichement abattus à l'abattoir de Kamboinsin. Ensuite, les femelles ont été triées puis enfin broyées légèrement dans un mortier en porcelaine. Le broyat a été filtrées avec des tamis de mailles décroissantes de $55 \mu \mathrm{m}$ et 25 $\mu \mathrm{m}$. La solution d'œufs obtenue a été réajustée à 100 œufs par millilitre.

\section{iii. Obtention et Récoltes des larves infestantes $\angle 3$}

Les larves L3 ont été obtenues en cultivant pendant 14 jours à $31^{\circ} \mathrm{C}$ les œufs dans des fèces d'ovins préalablement stérilisés à l'étuve à $130^{\circ} \mathrm{C}$ pendant 24 heures et mélangés avec de la sciure du bois. Après 14 jours la culture a été montée dans un gaze puis déposé sur le dispositif de Baermann pour récolter les larves L3. La solution larvaire obtenue a été mise dans un bécher et déposée au congélateur pendant 3 heures. Les larves ayant migrées au fond ont été récupérées et centrifugées trois fois à 2000 trs/mns pendant $10 \mathrm{mns}$. La solution larvaire a été ensuite réajustée à 2000 larves/ $\mathrm{ml}$.

\section{iv. Test d'inhibition de l'éc/osion}

Le test d'inhibition de l'éclosion a été réalisé selon la méthode modifié de Kaboré. (2009). $1 \mathrm{ml}$ de la solution larvaire réajustée à 100 œufs/ ml a été mise en contact avec $1 \mathrm{ml}$ de chaque concentration d'extraits dans une boîte de pétri $(60 \times 15 \mathrm{Cm})$ puis incubé 
pendant 48 heures à $27^{\circ} \mathrm{C}$. Après 48 heures quelques gouttes de lugol ont été ajoutées dans chaque boîte de pétri pour stopper l'évolution des œufs.

Le test a été répété trois fois avec trois réplicas pour chaque concentration par répétition.

Le nombre de larve et d'œufs non-éclos ont été évalués en déposant $50 \mu \mathrm{l}$ entre lame et lamelle et observant au microscope à l'objectif X10.

Le pourcentage d'inhibition de l'éclosion des œufs a été calculé selon la formule suivante (Kaboré, 2009):

Pourcentage d'inhibition $(\%)=100(1-X 1 / X 2)$

X1: Nombre d'œufs éclos dans les extraits testés

X2: Nombre d'œufs éclos dans le témoin négatif

\section{v. Test d'embryonnement}

Le test d'embryonnement a été réalisé selon la méthode modifiée de Waboponé et al. (2006) décrite par Kaboré. (2009). $1 \mathrm{ml}$ de la solution d'œuf à 100 œufs/ml a été incubé dans des boîtes de pétri (60X15 $\mathrm{Cm})$ à $27^{\circ} \mathrm{C} .24$ heures après, lorsque les larves L1 ont été transparentes et ont commencé à se mouvoir dans les enveloppes des œufs, $1 \mathrm{ml}$ de chacune des concentration test de chaque extrait a été ajouté dans chacune des boîtes de pétri constituant le lot test. $1 \mathrm{ml}$ du PBS $1 \mathrm{X}$ et $1 \mathrm{ml}$ de lévamisol à $2,5 \mathrm{mg} / \mathrm{ml}$ ont été ajouté dans les boîtes de pétri pour tenir respectivement de témoin négatif et témoin positif. Après 6 heures de contact des extraits et des œufs embryonnés quelques gouttes de lugol ont été ajoutées dans chaque boîte de pétri pour stopper l'évolution des œufs.
Le test a été réalisé en trois répétition avec 3 réplicas pour chaque répétition.

Le taux de paralysie larvaire (TPL) a été déterminé selon la formule de D'Angelo et al. (2014):

Nombre de Larves L1

$$
\mathrm{TPL}=100-\left[\left(\frac{)^{* 100}\right]}{\text { Nombre d'œufs embryonnés }}\right.\right.
$$

\section{vi. Test d'inhibition du dégainement larvaire}

Le test de dégainement des larves L3 a été effectué selon la méthode décrite par Par Zabré. (2018) combiné à celle de Okombé.(2011) et Marion.(2015).

En plus des trois concentrations $(3,12 \mathrm{mg} / \mathrm{ml}$; $6,25 \mathrm{mg} / \mathrm{ml} ; 12,5 \mathrm{mg} / \mathrm{ml}$ ) des extraits de chaque plante, et un témoin négatif (PBS) réalisées précédemment, une solution dite de Milton (javel 2,6\% dilué dans du $\mathrm{Nacl} 0,9 \%$ au 1/300) a été réalisée.

$1 \mathrm{ml}$ de la solution larvaire a été incubé avec 4 $\mathrm{ml}$ de la solution d'extrait pendant $3 \mathrm{~h}$ dans des boîtes de pétri $(60 \times 15 \mathrm{Cm})$ et agité chaque 30 minute. Après 3h, $200 \mu$ l ont été prélevés de chacune des boites de pétri et mis en contact avec $200 \mu \mathrm{l}$ de la solution de milton dans un tube eppendorf $2 \mathrm{ml}$. Le test a été répété 3 fois.

La cinétique de dégainement a été suivie au microscope X20 aux temps, T0, T20, T40 et T60mns après contact avec la solution de Milton en mettant $50 \mu \mathrm{l}$ entre et lamelle et en comptant les larves dégainées et engainées.

Le pourcentage des larves dégainées a été calculé selon la formule suivante:

Nombre de larves dégainées

Nombre total de larves

de l'évolution du dégainement des larves $L 3$ en fonction du temps.

\section{e) Analyse des données}

Les données ont été saisies sur le logiciel Excel 2016 qui a servi aux calculs des moyennes et Ecart Type. Les moyennes du taux d'inhibition des éclosions ainsi que celles du taux de paralysies larvaires et du dégainement larvaire ont été comparées à l'aide d'une analyse de variance (ANOVA) à une voie suivi d'une comparaison multiple de moyenne au seuil de $5 \%$ avec le Packages Rcmdr version 2.7-1 du logiciel $R$ version 4.1.0.

Une régression linéaire par la méthode probit a été effectuée avec le logiciel SPSS STATISTICA 26 afin de déterminer les concentrations inhibitrices 50\% de l'éclosion des œufs et celle qui engendre la paralysie larvaire des œufs embryonnées de 50\%.

Le logiciel PRISM GraphPad Software 5.00.288 nous a servi de tracer les graphiques doses-réponses 


\section{RÉSULTATS}

Tableau 1: Effet des extraits aqueux de Striga hermonthica (Del.) Benth et Cératothéca Sésamoides Endl sur l'éclosions des œufs de Haemonchus contortus

\begin{tabular}{|c|c|c|c|c|c|c|c|c|c|c|}
\hline \multicolumn{11}{|c|}{ Pourcentage d'inhibition de l'éclosion des CEufs } \\
\hline \multirow[b]{2}{*}{ Répétitions } & \multicolumn{5}{|c|}{$\begin{array}{c}\text { Doses }(\mathrm{mg} / \mathrm{ml}) / \text { Extrait aqueux du } S \\
\text { hermonthica(Del.) Benth }\end{array}$} & \multicolumn{5}{|c|}{$\begin{array}{c}\text { Doses }(\mathrm{mg} / \mathrm{ml}) / \text { Extrait aqueux } C \text {. } \\
\text { sésamoïdesEndl }\end{array}$} \\
\hline & 3,12 & 6,25 & 12,5 & $\mathrm{~T}(-)$ & $\mathrm{T}(+)$ & 3,12 & 6,25 & 12,5 & $\mathrm{~T}(-)$ & $\mathrm{T}(+)$ \\
\hline $\mathrm{R} 1$ & 23,08 & 46,16 & 38,46 & 0 & 53,84 & 1,86 & 22,22 & 50 & 0 & 48,16 \\
\hline $\mathrm{R} 1$ & 5,88 & 21,58 & 52,94 & 0 & 64,71 & 17,65 & 29,41 & 60,79 & 0 & 42,35 \\
\hline R3 & 15,38 & 23,08 & 30,76 & 0 & 53,84 & 21,05 & 22,81 & 50,88 & 0 & 78,95 \\
\hline Moyenne $\pm \boldsymbol{\sigma}$ & $\begin{array}{c}14,78 \\
\pm 8,61 \\
a b\end{array}$ & $\begin{array}{c}30,27 \\
\pm 13,78 \\
\text { bc }\end{array}$ & $\begin{array}{c}40,72 \\
\pm 11,26 \\
\text { cd }\end{array}$ & 0 & $\begin{array}{c}57,46 \\
\pm 11,26 \\
d\end{array}$ & $\begin{array}{c}13,52 \\
\pm 10,24 \\
a\end{array}$ & $\begin{array}{c}24,81 \\
\pm 3,99 \\
a\end{array}$ & $\begin{array}{c}53,89 \\
\pm 5,99 \\
b\end{array}$ & 0 & $\begin{array}{c}56,49 \\
\pm 19,67 \\
b\end{array}$ \\
\hline
\end{tabular}

R: Répétitions, $T$ (-): Témoin négatif (PBS: Phosphate-Buffered Saline1X), T(+): témoin positif (Lévamisole à 2,5 mg/ml), $\sigma$ : EcartType, $(a, b, c)$ différences entre les colonnes pour chaque extrait, les colonnes avec les lettres distincts ont les moyennes de pourcentage d'inhibition statistiquement différentes entre elles au seuil de 5\%.

Tableau 2: Valeurs des Concentrations entrainants l'inhibition de $50 \%$ d'éclosion des œufs obtenues à partir de l'équation de la droite de régression

\begin{tabular}{ccccc}
\hline & $\mathrm{R}^{2}$ d'ajustement & $\mathrm{a}$ & $\mathrm{b}$ & $\mathrm{Cl} 50$ \\
\hline C. sesamoïdesEndl & 0,97 & 2,15 & 1,99 & $11,83 \mathrm{mg} / \mathrm{ml}$ \\
\hline $\begin{array}{c}\text { S. hermonthica (Deli.) } \\
\text { Benth }\end{array}$ & 0,87 & 1,19 & 0,08 & $14,93 \mathrm{mg} / \mathrm{ml}$ \\
\hline
\end{tabular}

a: Ordonnée à l'origine b: pente de la droite régression, C150: Concentration qui inhibe 50\% de l'éclosion des œufs

Le tableau 1 montre l'effet des extraits aqueux des plantes testées sur l'inhibition de l'éclosion des œufs de $H$. contortus.

L'analyse de ce tableau nous indique que les extraits aqueux des plantes testées ont une activité inhibitrice de l'éclosion des œufs moyenne voire faible.En effet, on remarque une activité inhibitrice légèrement supérieure ou égale à $50 \%$ de l'éclosion des œufs seulement à la dose la plus élevée qui est de 12,5 $\mathrm{mg} / \mathrm{ml}$. Aussi seulement l'extrait aqueux de C. sesamoïdes Endl a obtenu un taux moyen d'inhibition de 53,89\% alors que celui de l'extrait aqueux du $\mathrm{S}$. hermonthica (Del.) Benth est de 40,72\%. Ce tableau nous indique aussi une faible activité inhibitrice du lévamisole (témoin positif) qui est de $56,49 \%$ en moyenne pour le test avec l'extrait aqueux de $C$. sesamoïdes Endl et 57,46\% en moyenne avec l'extrait aqueux du S. hermonthica (Deli.) Benth.

Le tableau 2 nous indique les valeurs de la droite de régression qui nous a permis d'obtenir les concentrations inhibitrices des extraits des deux plantes testées.

Tableau 3: Effet des extraits aqueux de Striga hermonthica (Del.) Benth et Cératothéca sésamoïdes Endl sur la paralysie des larves L1 embryonnées de Haemonchus contortus

Pourcentage de paralysie larvaire des CEufs embryonnés

\section{$\operatorname{Doses}(\mathrm{mg} / \mathrm{ml}) /$ Extrait aqueux du $S$ hermonthica(Del.) Benth \\ Doses $(\mathrm{mg} / \mathrm{ml}) /$ Extrait aqueux $C$. sésamoïdes Endl}

\begin{tabular}{ccccccccccc} 
Répétitions & $\mathbf{3 , 1 2}$ & $\mathbf{6 , 2 5}$ & $\mathbf{1 2 , 5}$ & $\mathrm{T}(-)$ & $\mathrm{T}(+)$ & 3,12 & 6,25 & 12,5 & $\mathrm{~T}(-)$ & $\mathrm{T}(+)$ \\
\hline R1 & 26,67 & 26,67 & 53,34 & 10 & 66,67 & 30 & 34,34 & 54,67 & 5 & 55 \\
\hline R1 & 20 & 20 & 46,67 & 10 & 60 & 36,67 & 32 & 60 & 0 & 60 \\
\hline R 3 & 33,34 & 33,34 & 53,34 & 15 & 73,34 & 26,67 & 35 & 67 & 10 & 80 \\
\hline Moyenne & 26,67 & 37,78 & 51,11 & 11,66 & 66,67 & 31,11 & 33,78 & 60,56 & 5 & 65 \\
$\mathbf{\pm \sigma}$ & $\pm 6,67$ & $\pm 3,8$ & $\pm 3,85$ & $\pm 2,88$ & $\pm 6,7$ & $\pm 5,09$ & $\pm 1,58$ & $\pm 6,12$ & \pm 5 & $\pm 13,23$ \\
& $\mathrm{~b}$ & $\mathrm{bc}$ & $\mathrm{c}$ & $\mathrm{a}$ & $\mathrm{d}$ & $\mathrm{b}$ & $\mathrm{b}$ & $\mathrm{c}$ & $\mathrm{a}$ & $\mathrm{c}$
\end{tabular}

R: Répétitions, $T$ (-): Témoin négatif (PBS : Phosphate-Buffered Saline1X), $T(+)$ : témoin positif (Lévamisole à 2,5 mg/ml), $\sigma$ : EcartType, $(a, b, c)$ : différences entre les colonnes pour chaque extrait, les colonnes avec les lettres distinctes ont les moyennes de pourcentage de paralysie larvaire statistiquement différentes entre elles au seuil de 5\%. 
Tableau 4: Valeurs des Concentrations entrainants la Paralysie larvaire de 50\% des œufs embryonnées obtenues à partir de l'équation de la droite de régression

\begin{tabular}{ccccc}
\hline & $\mathrm{R}^{2}$ d'ajustement & $\mathrm{a}$ & $\mathrm{b}$ & $\mathrm{Cl} 50$ \\
\hline C. sesamoïdesEndl & 0,94 & 0,84 & 0,09 & $9,80 \mathrm{mg} / \mathrm{ml}$ \\
\hline $\begin{array}{c}\text { S. hermonthica (Deli.) } \\
\text { Benth }\end{array}$ & 0,97 & 0,79 & 0,07 & $11,79 \mathrm{mg} / \mathrm{ml}$ \\
\hline
\end{tabular}

a: Ordonnée à l'origine b: pente de la droite régression, Cl50: Concentration qui entraine la paralysie larvaire de $50 \%$ des œufs embryonnées.

Le tableau 3 nous indique l'effet des extraits aqueux de S. hermonthica (Deli.) Benth et de C. sesamoïdes Endl sur la paralasie larvaire des œufs embryonnées de $H$. contortus.

Ce tableau nous montre que les extraits aqueux des deux plantes testées ont un taux moyen d'activité de paralysie larvaire des œufs embryonnées de $H$. contortus. En effet, ce tableau nous indique un taux moyen de paralysie larvaire de $51,11 \%$ et $60,56 \%$ respectivement pour l'extrait aqueux du $S$. hermontica (Deli.) Benth et de celui de C. sesamoides Endl.
Nous remarquons un taux de paralysie larvaire de $11,66 \%$ du lot témoin négatif (PBS1X) pour le test avec l'extrait aqueux du S. hermonthica (Deli.) Benth et $5 \%$ pour le test avec l'extrait aqueux de C. sesamoides Endl. Cependant, l'activité du témoin positif (lévamisole) n'a été que de $66,67 \%$ et $65 \%$ respectivement pour test avec S. hermonthica (Del.) Benth et C. sesamoides Endl. Le tableau 4 nous indique les valeurs de la droite de régression qui nous a permis d'obtenir les concentrations des extraits des deux plantes testées qui engendrent la paralysie larvaire de $50 \%$ des œufs embryonnés.

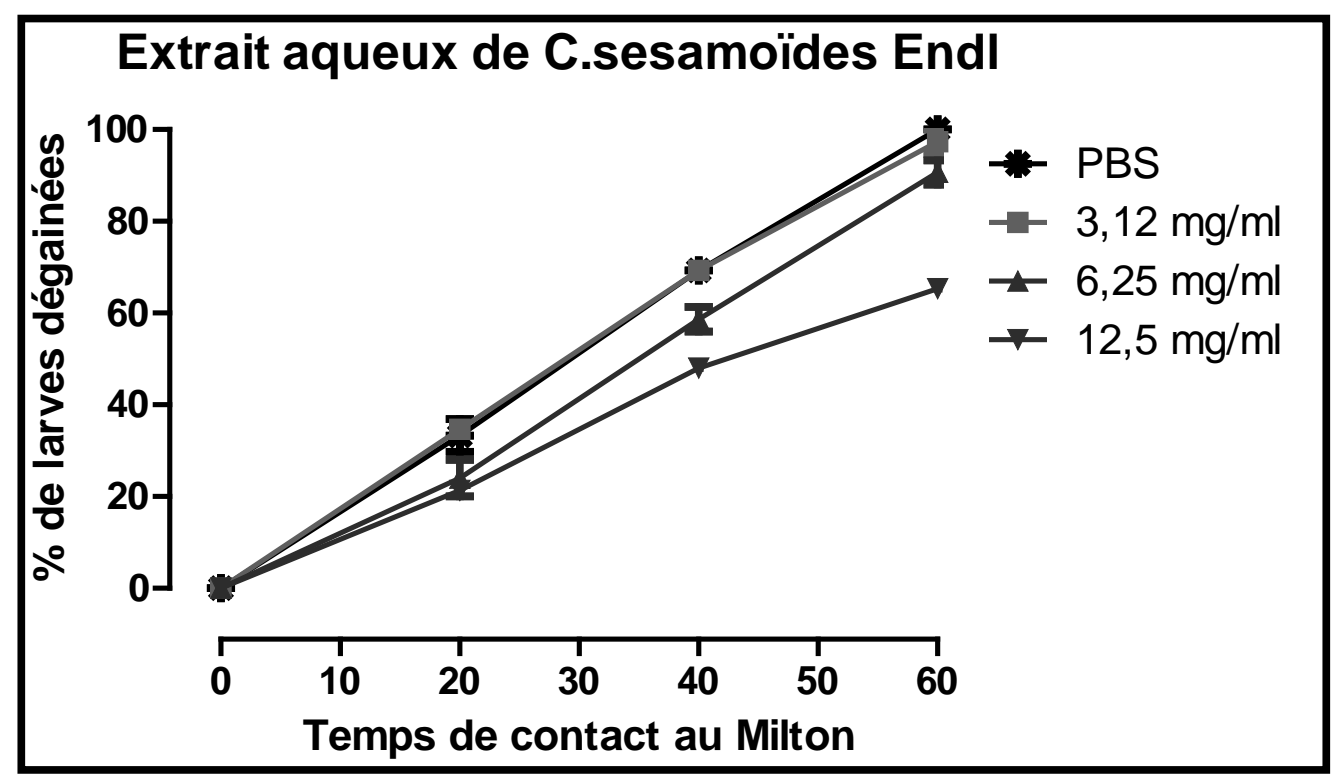

Figure 1: Evolution du dégainement des larves L3 avec l'extrait aqueux de Ceratotheca sesamoïdes Endl en fonction du temps

La figure 1 nous montre l'évolution du dégainement en fonction du temps après contact avec la solution de Milton des larves L3 mises en contact avec les différentes concentrations de l'extrait aqueux de C. sesamoïdes Endl. La figure 1 nous montre une évolution similaire des courbes du dégainement des larves mises en contact avec le témoin négatif (PBS 1X) et celles mises en contact avec la dose $3,12 \mathrm{mg} / \mathrm{ml}$. L'analyse de ce graphique nous indique aussi que les doses $6,25 \mathrm{mg} / \mathrm{ml}$ et $12,5 \mathrm{mg} / \mathrm{ml}$ de l'extrait aqueux de C. sesamoïdes Endl ont eu une inhibition de dégainement respectivement faible et moyenne des larves.
La comparaison multiple des moyennes du pourcentage de dégainement par la méthode de Tukeys montre une forte significativité entre la moyenne de pourcentage du dégainement des larves $L 3$ au temps (T60) et les temps (T0) ainsi qu'au temps (T20) avec une probabilité $p<0,001$ alors que la moyenne du pourcentage du dégainement des larves au temps (T60) et celle au temps (T40) n'a pas été très significative $p<$ 0,011 . 


\section{Extrait aqueux de S. hermonthica (Deli.) Benth}

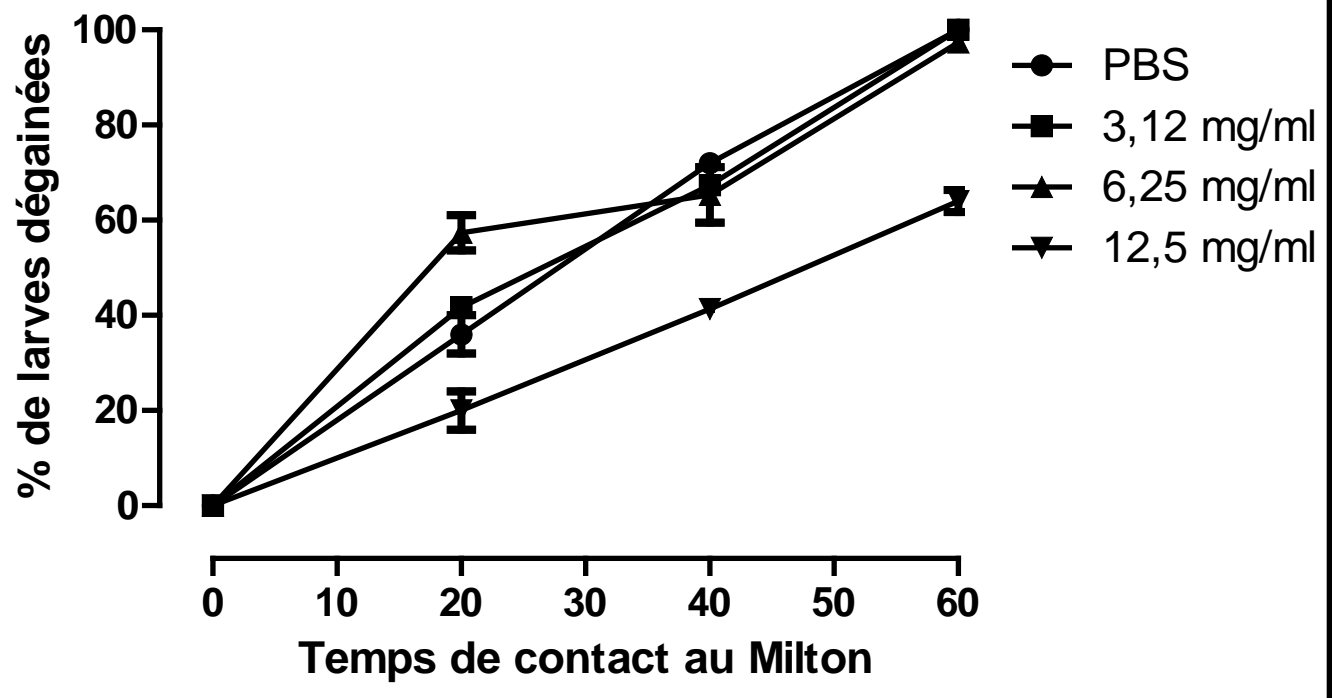

Figure 2: Evolution du dégainement des larves L3 avec l'extrait aqueux de Striga hermonthica (Deli.) Benth en fonction du temps

La figure 2 nous montre l'évolution du dégainement des larves L3 en fonction du temps après contact à la solution de Milton des larves L3 mises en contact avec les différentes concentrations de l'extrait aqueux du S. hermonthica (Deli) Benth. Ce graphique nous indique une évolution identique des courbes en fonction du temps de la moyenne de dégainement des larves L3 mises en contact avec les concentrations 3, 12 $\mathrm{mg} / \mathrm{ml}, 6,25 \mathrm{mg} / \mathrm{ml}$ et celle mises en contact avec le témoin négatif (PBS 1X).

La comparaison multiple des moyennes par la méthode de Tukey montre une forte significativité entre les moyennes du pourcentage de dégainement du temps (T60) et (T20) ainsi qu'aux temps (T60) et temps (T0) $\mathrm{P}<0,001$. Par contre les moyennes du pourcentage du déagienement du temps (T20) et temps (T0) ainsi que temps (T40) et temps (T60) n'ont pas été très significatifs avec respectivement $\mathrm{P}<0,011$ et $\mathrm{P}<$ 0,028 .

\section{Discussion}

Les résultats de cette étude nous montrent que l'inhibition de l'éclosion des œufs de $H$. contortus est doses-dépendante avec les extraits aqueux de C. sesamoïdes Endl et S. hermonthica (Deli.) Benth. En effet, l'extrait aqueux de C. sesamoïdes Endl, a obtenu un taux d'inhibition évolutif en fonction de la concentration qui atteint une moyenne de $53,89 \%$ à la dose de $12,5 \mathrm{mg} / \mathrm{ml}$. L'extrait aqueux du $\mathrm{S}$. hermonthica (Deli.) Benth a aussi eu un effet inhibiteur évolutif en fonction de la concentration mais de moyenne faible n'atteignant pas $50 \%$ à la dose de $12,5 \mathrm{mg} / \mathrm{ml}$. Par contre les extraits des deux plantes testées ont eu un effet inhibiteur et évolutif en fonction de la concentration qui atteint respectivement $51,11 \%$ pour S. hermonthica (Deli.) Benth et $60,56 \%$ pour C. sesamoïdes Endl. Koffi et al. (2018), ont eu des résultats similaires avec les extraits hydro-méthanoliques à $2000 \mu \mathrm{g} / \mathrm{ml}$ de sept (07) espèces parmi huit (08) dont seulement l'extraits hydrométhanoliques du Morus mesozyna a eu un de taux d'inhibition de l'éclosion de $84,08 \%$ alors que les sept (07) autres plantes ont eu des taux faibles allant de $5,65 \%$ avec Ficus lutea à 28, 08\% pour Antiaris africana. Cette dissemblance de résultats avec l'extrait du Morus mesozyna serait due à la nature du solvant d'extraction car les métabolites sécondaire extraits des plantes dépendent du solvant d'extraction. Ainsi, l'extraction aqueuse de nos plantes testées pourrait ne pas contenir les métabolite sécondaire pouvant inhiber l'éclosions des œufs jusqu'à un tel pourcentage que celui du Morus mesozyna. Par contre le fait que les sept (07) autres plantes testées par ces auteurs n'ont pas eu $50 \%$ d'inhibition de l'éclosions pourrait aussi s'expliquer par la nature leurs solvant d'extractions et aussi par la résistance à des œufs de $H$. contortus qui sont naturellement très résistante. D'Angelo et al. (2014) ont aussi eu des résultats différents au notre avec les extraits methylene Chloro-méthanolique de l'écorcé du tronc de Annona senegalensis à différentes doses sur Heligmosomoïdes Bakeri. En effet, ces auteurs, ont obtenu un taux d'inhibition de l'éclosion des œufs de $16,10 \%$ alors que le taux de paralysie embryonnaire des œufs embryonnées était de $20,80 \%$ à la dose la plus élevée qui est de $5000 \mu \mathrm{g} / \mathrm{ml}$. Cette différence de résultats serait due à la différence de l'espèce de nématode étudiée car notre présente étude porte sur $H$. contortus alors que leur étude à porte sur 
Heligmosoïdes Bakeri. Aussi, cette différence pourrait être due à la différence des plantes utilisées et à la différence des solvants d'extraction qui favorisent la présence de certains métabolites secondaires que d'autres. Les extraits des feuilles de A. leiocarpus et des écorces du tronc de $D$. oliveri ont eu un effet dosedépendant sur la paralysie des œufs embryonnés avec respectivement une Cl50 de $411,4 \mu \mathrm{g} / \mathrm{ml}$ pour $A$. leiocarpus et $362,3 \mu \mathrm{g} / \mathrm{ml}$ pour $D$. oliveri et une inhibition de l'éclosion des œufs de 100\% pour les extraits aqueux de ces deux plantes (Kaboré, 2009). La différence d'efficacité de ces plantes avec celles des plantes utilisées dans notre présente étude pourrait être due à la nature des plantes utilisées. En effet, notre présente étude a porté sur des herbacées entières alors que cette étude a porté sur des feuilles et écorces de ligneux.

Cette présente étude nous montre à travers les résultats obtenus que les extraits aqueux de $C$. sesamoïdes Endl et ceux du S. hermonthica (Deli.) Benth ont une inhibition qui est dose-dépendante sur le dégainèrent des larves L3 de $\mathrm{H}$. contortus. En effet, les figure 1 et 2 nous montrent une évolution similaire de la cinétique du dégainement des larves mises en contact avec le témoin négatif (PBS1x) et celles mises en contact avec la dose de $3,12 \mathrm{mg} / \mathrm{ml}$. Par contre l'évolution des courbes des larves mises en contact avec le témoin négatif (PBS1x) et celles des larves mises en contacts avec la dose de $3,12 \mathrm{mg} / \mathrm{ml}$ est différente de celle des larves mises en contact avec la doses de $6,25 \mathrm{mg} / \mathrm{ml}$ et de celles des larves mise en contact avec la doses de $12,5 \mathrm{mg} / \mathrm{ml}$ dont l'évolution de l'inhibition du dégainement est juste au-dessus de $50 \%$. Les extraits aqueux et méthanolique d'Acacia nilotica ainsi que $A$. raddiana ont obtenu une inhibition du dégainement des larves L3 mise en contact avec les doses de $1,2 \mathrm{mg} / \mathrm{ml}$ et $0,6 \mathrm{mg} / \mathrm{ml}$ qui atteint $100 \%$ d'inhibition (Zabré, 2018). La différence avec les résultats obtenus dans cette présente étude pourrait être due à la nature des plantes utilisées car notre présente étude a porté sur les herbacées entières alors qu'il s'agit des feuilles de ligneux dans l'autre étude.

Cette efficacité moyenne des extraits aqueux de C. sesamoïdes Endl et S. hermonthica (Deli.) Benth serait due à la présence de certains métabolites secondaires tels que les tanins et les flavonoïdes dans ces extraits aqueux. Cependant au vu de résultats d'inhibition de l'éclosion des œufs frais et des œufs embryonnés (paralysie larvaire) ainsi que celui du dégainement des larves L3 nous pouvons émettre I'hypothèse que la solution aqueuse ne permet pas d'extraire la dose suffisante à inhiber l'évolution des œufs et des larves à 100\%.

\section{Conclusion}

La présente étude confirme que les extraits aqueux de C. sesamoïdes Endl, et S. hermonthica (Deli.)
Benth contiennent des composés bioactifs qui inhibe l'évolution des œufs et le dégainement des larves $\mathrm{L} 3$ de $H$. contortus. Cependant, du fait que l'inhibition de l'évolution des œufs et du dégainement des larves $L 3$ n'atteint pas $100 \%$ nous laisse penser que la présence de ces composés bioactifs serait optimisée par un autre solvant d'extraction. Toutefois les extraits aqueux de $C$. sesamoides Endl et $S$. hermonthica (Deli.) Benth pourraient être employés comme désinfectant sur les pâturages pour interrompre le cycle évolutif des nématodes et diminuer ainsi la charge parasitaire des pâturages.

\section{Bibliographie}

1. Adama Kabore, 2009: Activités anthelminthiques de deux plantes tropicales testées in vivo et in vitro sur les strongles gastro-intestinaux des ovins de races mossis du Burkina Faso. Thèse de doctorat unique en développement rural, Université polytechnique de Bobo-Dioulasso. 167p.

2. Alain Gérard Ambroise Apala, Amoin Marie Amélie Clarisse Komoin-Oka, Kouakou Rufin Assare, Gédéon Amian, Kouakou Eliezer N'Goran, 2020: Efficacité des anthelminthiques usuels contre les strongles digestifs chez les ovins au centre de la Côte d'ivoire.Int. J. Biol. Chem. Sci. 14(2): 378-389, 2020.

3. Francesco D'Angelo, WaboPoné J, Yondo Jeannette, Komtangi Marie Claire, SauroVittori, MpoameMbida. 2014: Evaluation of Ovicidal and Larvicidal Activities of Methylene Chloride-Methanol Extract of Annona Senegalensis (Annonaceae) Stem Barkon Heligmosomoides Bakeri (Nematoda, Heligmosomatidae). Global Journal of Science Frontier Research: C Biological Science Volume 14 Issue 2 Version 1.0 Year 2014.

4. Gertrude Mbogning Tayo, Josué Wabo Poné*, Marie Claire Komtangi, Jeannette Yondo, Alidou Marc Ngangout, Mpoame Mbida, 2014: Anthelminthic Activity of Moringao leifera Leaf extracts evaluated in Vitro on four developmental stages of Haemonchus contortus from goats. American Journal of Plant Sciences, 2014, 5, 1702-1710.

5. Hafidh Akkaria, Jed Jebali, Mohamed Gharbia, Moez Mhadhbia, Soufia Awadic, Mohamed Aziz Darghoutha, 2013: Epidemiological study of sympatric Haemonchus species and genetic characterization of Haemonchus contortus in domestic ruminants in Tunisia. Veterinary Parasitology 193 (2013) 118- 125.

6. J. Charlier, E. R. Morgan, L. Rinaldi, J. van Dijk, J. Demeler, J. Höglund, H. Hertzberg, B. Van Ranst, G. Hendrickx, J. Vercruysse, F. Kenyon, 2014: Practices to optimise gastrointestinal nematode control on sheep, goat and cattle farms in Europe using targeted (selective) treatments. Veterinary Record (2014), 250-255. 
7. J.M. Burke, R.M. Kaplan, J.E. Miller, T.H. Terrill, W.R. Getz, S. Mobini, E. Valencia, M.J. Williams, L.H. Williamson, A.F. Vatta, 2007: Accuracy of the FAMACHA system for on farm use by sheep and goat producers in the southeastern United States. Veterinary Parasitology 147 (2007) 89-95.

8. Koffi Yao Mesmin, Adama Bakayoko, Gervaise AmoinKouame, Mamidou Witabouna Koné, 2018: Activité Anthelminthique In Vitro Et Teneurs En Tanins Et Flavonoïdes De Huit Plantes Fourragères Utilisées En Elevage Des Petits Ruminants En Côte d'Ivoire. European Scientific Journal 2018 Vol.14, No.15: $1857-7881$.

9. Marion Peters, 2015: Etat des lieux des traitements anthelminthiques sur Haemonchus contortus, strongle gastro-intestinal des ovins. Evaluation chimique et biologique sur ce parasite de deux macromycètes: Phellodon niger et Hydnellum ferrugineum Doctorat d'Etat en Pharmacie, Université Angers. 107p.

10. N. Maingi, R.C. Krecek, N. van Biljon, 2006: Control of gastrointestinal nematodes in goats on pastures in South Africa using nematophagous fungi Duddingtonia flagrans and selective anthelmintic treatments. Veterinary Parasitology 138 (2006) 328-336.

11. V.F.G.N. Dedehou, P.A. Olounladé, A.D. Adenilé, E.V.B. Azando, G.G. Alowanou, F.D. Daga, M.S. Hounzangbé-Adoté, 2014: Effets in vitro des feuilles de Pterocarpus erinaceus et des cosses de fruits de Parkia biglobosa sur deux stades du cycle de développement de Haemonchus contortus nématode parasite gastro-intestinal de petits ruminants. Journal of Animal and Plant Sciences, 2014. Vol.22, Issue 1 : 3368-3378.

12. Victor Okombe Embeya, 2011: Activité antihelminthique de la poudre d'écorce de racine de Vitex thomasii De Wild (Verbenaceae) sur Haemonchus contortus chez la chèvre. Doctorat de médecine vétérinaire et santé animale. Université de Lubumbashi. 258p

13. W. Mamidou Koné, K. Kamanzi Atindehou, Traore' Dossahoua, Bruno Betschart, 2005: Anthelmintic Activity of Medicinal Plants Used in Northern Côte D'Ivoire Against Intestinal Helminthiasis. Pharmaceutical Biology, 2005, Vol. 43, No. 1, pp. 72-78.

14. Zabré Genevieve, 2018: Qualités antiparasitaires et nutritionnelles de Acacia niloticavar adansonii (Guill. et Perr.) O. Ktze et de Acacia raddiana(Savi) Brenan, deux plantes fourragères sahéliennes du Burkina Faso. Thèse de Doctorat unique en sciences biologiques et appliquées/ Physiologie et santé animale, Université Ouaga I Pr. Joseph KI-ZERBO. $142 p$ 